\title{
DERMAL THERMO-PORATION WITH A PDMS-BASED PATCH FOR TRANSDERMAL BIOMOLECULAR DETECTION
}

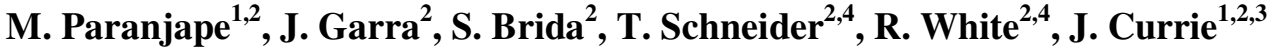 \\ ${ }^{1}$ Department of Physics, Georgetown University, Washington D.C. 20057 \\ ${ }^{2}$ Georgetown Advanced Electronics Laboratory (GAEL), Washington D.C. 20057 \\ ${ }^{3}$ Department of Pharmacology, Georgetown University, Washington D.C. 20057 \\ ${ }^{4}$ Science Applications International Corporation (SAIC), McLean, VA 22102
}

\begin{abstract}
This paper describes the fabrication of an adhesive bandage, consisting of a stack of compliant polydimethylsiloxane (PDMS) microfluidic elements, to perform controlled and non-invasive transdermal (through the skin) sampling of bio-molecules. The patch-like device will be worn on the skin, and has two PDMS component layers that form vertically oriented micro-fluidic channels and reservoirs. In addition, micro-heaters are integrated onto the PDMS layer that will be in contact with the skin, and are used sequentially to thermally ablate tiny micro-pores through only the dead skin layer, allowing for easier diffusion of the normally trapped bio-molecules to the skin surface. The microchannels and fluid-filled reservoirs assist in transporting these biomolecules from just beneath the dead skin layer to a colorimetric absorbent paper detector situated on top of the bi-layer PDMS patch. This paper will focus on the fabrication of the prototype PDMS patch. A brief introduction to the operation of the patch microsystem is first presented.
\end{abstract}

\section{INTRODUCTION}

A means of non-invasive and real-time sampling of specific bio-molecules from the interstitial fluid would provide an important and convenient means for monitoring individuals prone to high health risks, such as those exposed to environmental, chemical, or biological hazards, have predisposed health conditions, or need to be closely monitored following illness. The polydimethylsiloxane (PDMS) based skin patch being presented in this paper is a novel bioMEMS device relying on the advances made in microfabrication technologies related to both PDMS and thick SU-8 photoresist mold processing [1-3]. The prototype of the PDMS patch will be used to sample glucose however the development of this microsystem will provide an enabling technology for transdermal sampling of other bio-molecules that do not normally diffuse across the skin [4]. Due to the large size of many bio-molecules of medical relevance, they are unable to penetrate the densely packed layers of dead skin (stratum corneum, or SC) cells to reach the skin surface. The dead skin layer, in an unbroken state, acts as a very effective seal against interstitial fluid leakage and bio-molecular containment. When the seal is compromised, the interstitial fluid and the biomolecules contained therein become available on the skin surface.

The method by which large bio-molecules, such as glucose, can reach the skin surface for subsequent analysis by the PDMS patch is by opening a microscopic region of the stratum corneum to allow the glucose (and other bio-molecules) to diffuse naturally to the skin surface. By locally applying a high temperature in proximity to the skin surface, the dead skin cells can be thermally ablated, or vaporized, using micro-heaters integrated on the PDMS layer closest to the skin surface. By regulating the current through a resistive heater and its time duration, the generated heat pulse can be controlled to such an extent that neither living tissue nor nerves are affected by the momentary elevation in temperature.

A cross-sectional view of the PDMS adhesive patch is shown in Fig. 1, comprising of a micro-fluidic sampling system coupled with a thermal ablation system allowing body analyte sampling at the Stratum Corneum (SC)/Viable Epidermis (VE) interface without invasive extraction of interstitial fluid. Upon creation of a micro-pore opening in the dead skin layer (SC), perfusion of the bio-molecules will be used in a non-inflammatory collection strategy. On-patch reservoirs encapsulating physiological fluid are used for bio-molecule perfusion, while capillary channels enable fluid transfer between the skin surface and the glucose detector. These components are illustrated in Fig. 1 (not to scale), showing three separate analysis systems, each in different states of transdermal transfer operation, described later. Each analysis system can be addressed individually and on-demand, as dictated by the use of the PDMS patch.

From Fig. 1, the microfluidic system can be viewed as three distinct PDMS levels rather than two: 1. the capillary level that forms the reservoir and transport capillaries, 2. the dermal interface, which provides the bottom seal to the reservoir and contains the thermal micro-ablation heaters, and 3. the detector interface that is in contact with the detector patch and provides the top seal for the reservoir. The operation of the device and the function of each level will be described in the following sections.

\section{PDMS PATCH OPERATION}

The PDMS-based patch is a macro-sized device approximately $1 \mathrm{~cm}^{2}$ in dimension, containing 25 individually addressable analysis systems for programmed or on-demand use. Both back and front sides of the prototype biomolecular detection bandage have been shown in Figs. 2 and 3. The colorimetric detection patch, produced by Dermal Systems International (DSI, San Francisco, CA) situated above the capillary channels changes color depending on the concentration of glucose being sampled. A representative color dot (boxed area of Fig. 3) has been magnified in Fig. 4. Additionally, the gray-scale gradient with corresponding glucose concentrations has been given.

The patch operates at the micron level to transport and detect bio-molecules from the host's interstitial fluid. This is accomplished by enhancing transdermal transfer of bio-molecules to a physiological sampling fluid, which is released by the PDMS patch during a sampling operation. The physiological fluid is normally encapsulated in a reservoir but is released using a bubble drive mechanism through electrolysis that forces this liquid out of the reservoir. The physiological fluid bathes the ablated micropore region and the bubble-driven fluid, in conjunction with capillary action, allows for fluidic mobility towards the glucose detector. Fig. 1 depicts the three possible (controllable) states of the individual micro-capillary systems. The leftmost microcapillary system (\#1) shows an exhausted capillary-reservoir pair that has already been used for an analysis procedure. The SC has 


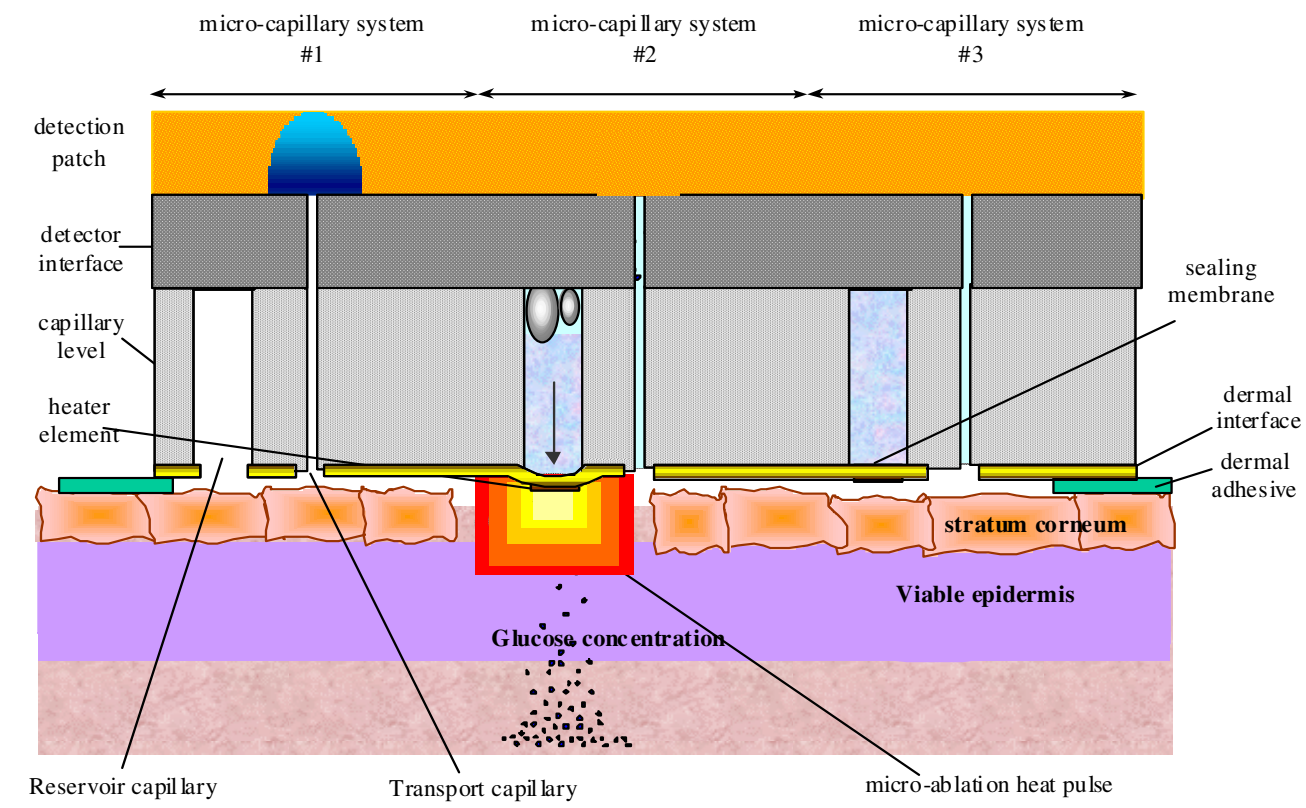

Fig. 1. The PDMS-based patch prototype located on the skin surface showing the Stratum Corneum (SC) and Viable Epidermis (VE). The microfluidic system is separated into three levels comprising the capillary level, the dermal interface, and the detector interface. The topmost layer uses colorimetric glucose detection paper. The figure also illustrates a concentration gradient of glucose.

restored itself some time after thermal ablation, the reservoir has emptied, and capture of glucose from the interstitial fluid has occurred, indicated by the stain on the glucose detector paper on the upper surface of the chip. The middle micro-capillary system

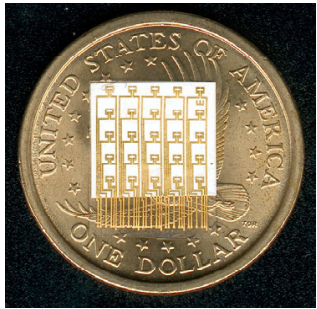

Fig. 2. PDMS patch prototype (backside)

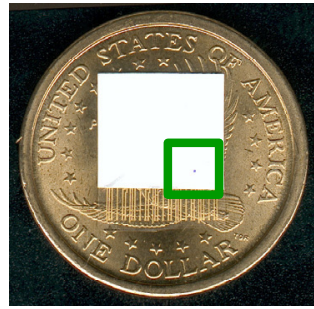

Fig. 3. PDMS patch prototype (topside)
(\#2) is performing an on-demand analysis. The rightmost capillary system is ready for future use. The remainder of the paper is dedicated to the fabrication process involved in producing the bi-layered PDMS patch.

\section{PDMS PATCh DESIGN CONSIDERATIONS}

PDMS technology makes use of standard silicon-based processing yet allows for rapid, cost effective fabrication of microfluidic systems that are flexible and compliant. This is an important design consideration for a dermal patch. Furthermore, by using the transparent PDMS, alignment of the two-layer patch becomes easier using standard optical microscopes and visually aligning each level independently. The PDMS (Dow Corning, Midland, MI) structures are created using SU-8 (MicroChem Corp. Newton, MA) as the mold form. In the design of the patch, the thickness of the PDMS for both the capillary/dermal layer and detector interface is nominally $200 \mu \mathrm{m}$. All metal electrodes, interconnects, bonding pads and heating elements are deposited directly on the PDMS surface and patterned using sputter deposition and a selective etch [5].

To facilitate ease in processing the PDMS layers, the silicone was molded onto SU-8 on glass handling-wafers. These handle wafers allow the PDMS to be placed in standard fabrication and test equipment, and more importantly, allows for simple alignment of the two levels required in the patch design. Therefore, when bonding multiple layers of PDMS together using oxygen plasma

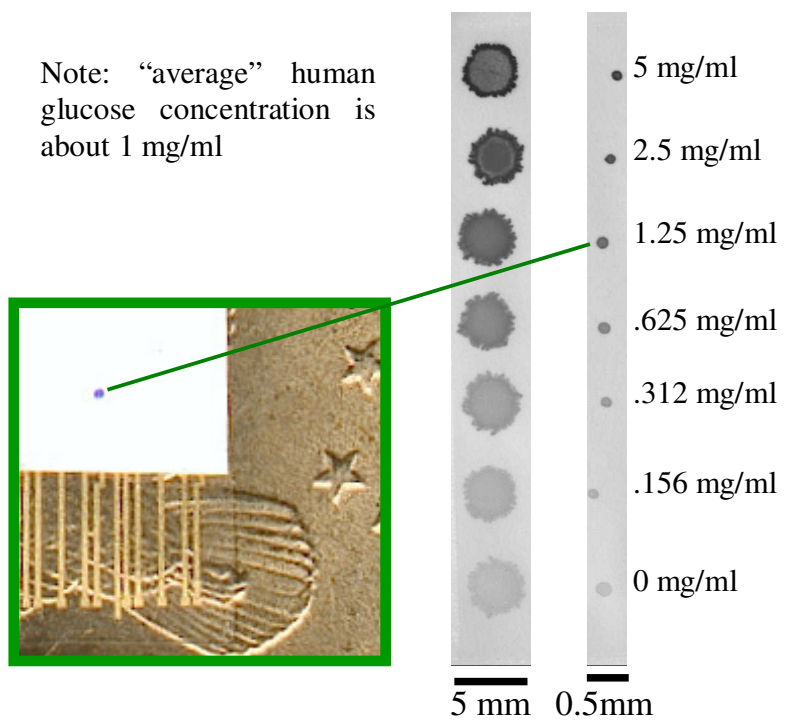

Fig. 4. Colorimetric detector patch that gives the glucose concentration.

treated surfaces [6], it is imperative that one handle wafer remains attached to the PDMS structure. Upon completion of the final device, the last step would be to release the multi-level PDMS 
stack from the remaining handle wafer. In order to process a particular side of a PDMS layer, a strategy was developed to allow for the selective removal of only one handle wafer over another. Otherwise, during a release procedure, no control would exist with regards to which handle wafer is removed. The basis of the selective release mechanism relies on a thin sacrificial layer being deposited directly onto the SU-8 mold prior to forming the PDMS structure [7]. Selective release is accomplished by dissolving only the sacrificial layer in a chemical etchant that does not attack any other sacrificial layer nor the structural PDMS and metalization.

\section{FABRICATION}

Microfluidic channels are typically formed in PDMS by casting a relatively thick layer of uncured polymer in a patterned SU-8 mold, as shown in Fig. 5. The SU-8 heights can range from $50-200 \mu \mathrm{m}$, while the PDMS is usually much thicker. The channels formed in this way direct fluid flow along the surface plane of the PDMS.

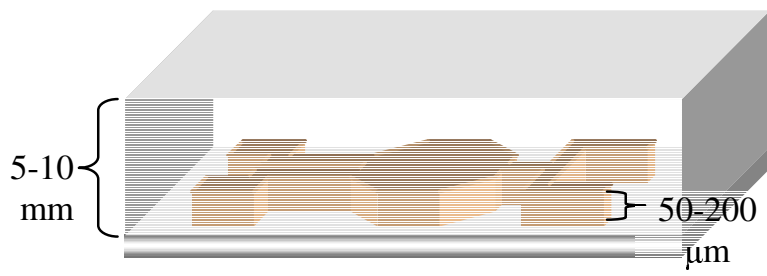

Fig. 5. Thick PDMS layer cast in an SU-8 mold for a typical microfluidic application with channels on the surface of the PDMS.

For the PDMS patch, precisely defined vertical capillary microchannels and fluid reservoirs need to be formed through the entire thickness of the PDMS layer, as shown in Fig. 6. This requires inserting the handle wafer, mold, and un-cured silicone elastomer into a specially devised mechanical jig that ensures planarity of the PDMS surface while removing excess elastomer so that the cured PDMS thickness is approximately equal to the mold relief height of the SU-8.

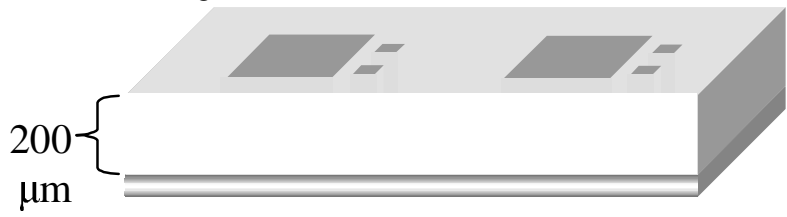

Fig. 6 PDMS cast in the SU-8 mold with vertical microchannels. The large reservoir is $500 \mu \mathrm{m} \times 500 \mu \mathrm{m}$ while the two smaller capillary channels are $100 \mu \mathrm{m} \times 100 \mu \mathrm{m}$. The small capillaries are separated by $100 \mu \mathrm{m}$ from each other, and from the large reservoir.

A limitation to the mechanical jig, which presses the PDMS and mold tightly together, is that it always tends to leave behind a thin residual PDMS layer on top of the mold features, which effectively seals the top end of the channels formed by the mold. Increasing the applied force can effectively eliminate the residual layer, but too much pressure can cause the finer mold features to fracture and become lodged in the PDMS layer upon release from the mold. The layer can also be etched away in a solution of tetrabutylammonium fluroride in n-methyl-2-pyrrolidinone [8]. For the PDMS patch, RIE processing was used [3] to selectively remove the residual sealing layer above the capillary mold feature while leaving the seal intact above the reservoir region. Thus, the limitation of the press has, in fact, become an advantage since it offers a means to have the reservoir sealed on one end while rendering fluidic-vias (or $f$-vias) after dry etching the capillary region. Fig. 7 presents an optical picture and an SEM micrograph of the reservoir sealed on one side and the $f$-via capillaries running through the $200 \mu \mathrm{m}$ thick PDMS after the RIE process.

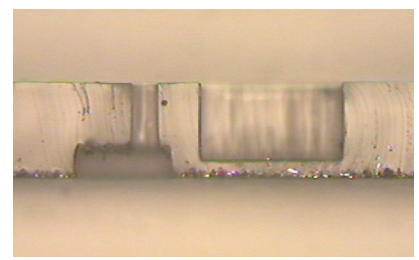

(a)

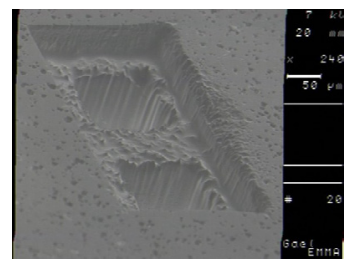

(b)
Fig. 7. (a) Cross-section of the PDMS layer showing the sealed reservoir on the right and the f-via capillary on the left. The dry etched region was designed to be larger than the capillary dimension, and (b) SEM showing PDMS texture after RIE processing and the oversized etch area with respect to the f-vias.

The process flow for the two levels of the PDMS patch has been illustrated in Figs. 8 and 9. The former figure illustrates the processing on the two separate layers while the latter shows the process on the bonded microsystem. The required bonding operation of the two individual PDMS layers is accomplished by exposing the two surfaces to an oxygen plasma. In order to avoid instantaneous bonding without proper alignment, methanol is used as a surfactant to allow optical alignment of the two layers. Once aligned, the methanol is evaporated by elevated temperature [9]. The process flow does not illustrate the selective removal of the glass handle wafers nor are the release layers shown. Information on these techniques can be found in [7].

\section{DISCUSSION}

The two-level PDMS patch was fabricated in a $5 \times 5$ array yielding 25 individually addressable sampling cells in a $1 \mathrm{~cm}^{2}$ area. Since this is only a prototype device, the dimensions of capillaries, reservoirs, and metalization traces were not minimized.

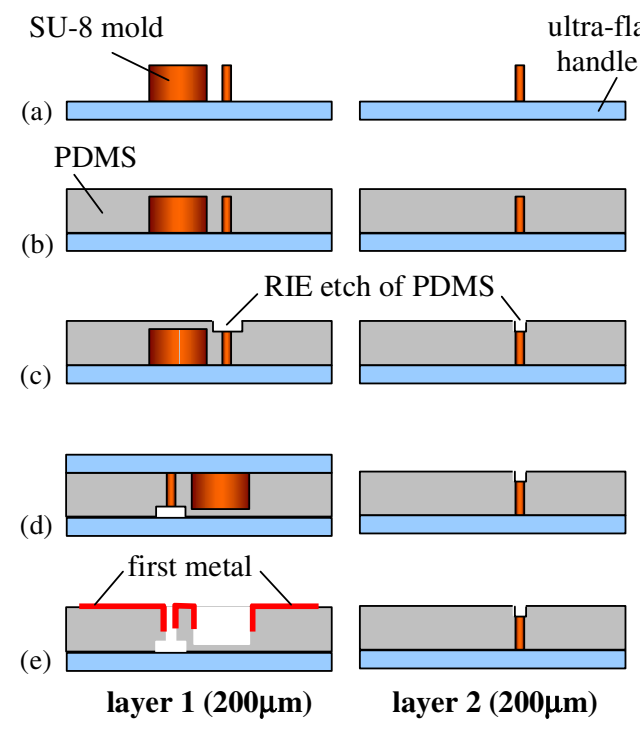

Fig. 8. (a) Original three-level (no RIE) SU-8 mold set made on glass substrates, with heights indicated. (b) Cast PDMS in SU-8 molds. (c) Perform selective RIE to open holes in capillary regions only. (d) Bond glass substrate to top of layer 1 and turn device upside down to selectively release other glass substrate. (e) Removal of glass substrate and SU-8 from layer 1 followed by metalization of layers 1 and 2 for interconnect. 
(a)

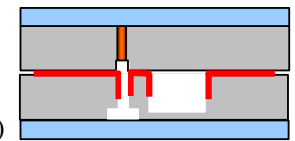

second metal

(b)

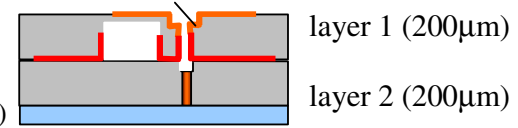

(c)

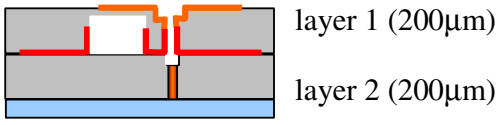

Fig. 9. (a) Layer 2 capillary is aligned and bonded onto layer 1. (b) Turning device upside down, glass substrate from layer 1 is released and metal traces deposited, including ablation micro-heaters. (c) Re-invert to perform final glass substrate release from layer 2 .

Although fabricating bioMEMS or microfluidic systems in PDMS is a relatively simple process with fast turnaround times and low material costs, there are some limitations in working with this material. Literature suggests that typical applications of PDMS are those when routing relatively large microfluidic channels parallel to the silicone surface, as was shown in Fig. 5. In these cases, any internal stresses that may be present in the PDMS, either during curing or after release from the SU-8 mold, are not critical. In the case of the PDMS patch, stress is an important factor for two important reasons. First, since the patch is a bi-layer, any stresses must be accounted for in view of the alignment of the two layers. Secondly, the PDMS has metalization traces that should not be compromised or deformed during the curing phase of the process.

The problem of PDMS stress has produced several fabrication challenges, but most problematic is that PDMS shrinks as it is cured into a solid state. When the silicone material is cast in an SU-8 mold, internal stresses build during the curing process. The polymer wants to shrink, but it remains stretched and held in place by the SU-8 features and by its attachment at the interface to the mold substrate. Once released, the polymer is allowed to relax and contract. This is a problem for the batch fabrication of devices in PDMS because the distance between cells or individual devices decreases when the layer is released from the mold. Subsequent processing steps including photolithographic patterning of metals and alignment to other device layers is impossible because the interdevice spacing has changed. Furthermore, the stresses in the PDMS layer are not necessarily isotropic, so shrinkage percentage can vary depending on the direction

As shown in Figs. 9 and 10, the patch prototype fabrication sequence required metalization and lithography to be performed after releasing the PDMS from the mold since the process required access to the sidewalls of the microchannel features. Metalization on a tensile PDMS substrate that is still in its mold can be problematic due to difficulties with adhesion, so the surface must be treated prior to deposition [5]. However, when the PDMS is finally released from the SU-8 at the end of the fabrication process, it relaxes, and the metal is subjected to strong compressive stress. The PDMS contracted as expected and more often than not, caused the metal pattern to be compromised. This buckling often caused the metal traces to detach at least partially from the PDMS substrate despite the good adhesion observed in the metal film after deposition.

\section{CONCLUSIONS}

A fabrication process for a bi-layer PDMS patch was developed for use in a dermal thermo-poration microsystem for transdermal sampling of bio-molecules. PDMS allows for rapid prototyping of compliant devices, ideal for an adhesive bandage. A $5 \times 5$ array of sampling cells was fabricated and tested for thermal characterization of the micro-ablation heater. Tests were conducted on surrogate (cloned) human skin, which mimics real skin in many ways including the stratum corneum thickness. Some limitations of the PDMS process include its shrinkage after removal from the SU-8 mold, resulting in misalignment and registry issues throughout the array. The compressive stresses also caused metal adhesion problems, metal and standard photoresist cracking, and PDMS delamination. Some of these problems may be resolved in the second iteration where PDMS will be replaced by silicon or SU-8 as a structural material. Such a solution may render a patch that is not as flexible as PDMS.

\section{ACKNOWLEDGMENTS}

The authors would like to thank Mr. Paul Goldey and Mr. Leon Der for their invaluable assistance in device fabrication and technical support. The assistance and contributions by the DSI staff is also gratefully acknowledged. This work is supported through a BioFlips DARPA-MTO contract (BAA 00-11, Program Manager: Dr. Michael Krihack).

\section{REFERENCES}

1. Y. Xia, G. M. Whitesides, "Soft Lithography", Angew. Chem. Int. Ed. Engl., 37, pp. 550-575, 1998.

2. D. C. Duffy, J. C. MacDonald, O. Schueller, G. M. Whitesides, "Rapid Prototyping of Microfluidic Systems in Polydimethylsiloxane", Anal Chem, 70, pp. 4974-4984, 1998.

3. J. Garra, T. Long, J. Currie, T. Schneider, R. White, M. Paranjape, "Polydimethylsiloxane (PDMS) Dry Etching for the Fabrication of Fluidic Microsystems", Journal of Vacuum Science Technology - A, to be published.

4. M. Paranjape, J. Currie, C. Peck, T. Schneider, R. White, "The B-FIT Microsystem Concept", BioMEMS and Biomedical Nanotechnology World 2000 Conf., Columbus, OH, 2000.

5. J. Garra, S. Brida, T. Schneider, J. Currie, M. Paranjape, "Metal Deposition on PDMS and SU-8 Surfaces", $2^{\text {nd }}$ DARPA Annual Progress Rep., 2001.

6. B. H. Jo, L. M. V. Lerberghe, K. M. Motsegood, and D. J. Beebe, "Three-dimensional Micro-channel Fabrication in Polydimethylsiloxane (PDMS) Elastomer", Journal of Microelectromechanical Systems, 9, pp. 76-81, 2000.

7. C. Luo, J. Garra, M. Cheng, S. Brida, T. Schneider, R. White, J. Currie, M. Paranjape, "Sacrificial Release Layers for PDMS and SU-8 Structures", $2^{\text {nd }}$ DARPA Annual Progress Rep., 2001.

8. S. Takayama, E. Ostuni, X. Qian, J. C. MacDonald, X. Jiang, M. Wu, P. Leduc, D. E. Ingber, G. M. Whitesides, "Patterning the Topographical Environment for Mammalian Cell Culture Using Laminar Flows in Capillaries", IEEE-EMBS Special Topic Conference on Microtechnologies in Medicine and Biology, Lyon, France, pp. 322-325, 2000.

9. J. A. Davis, S. Raty, D. T. Eddington, I. K. Glasgow, H. C. Zeringue, M. B. Wheeler, D. J. Beebe, "Development of Microfluidic Channels for the Culture of Mammalian Embryos", IEEE-EMBS Special Topic Conference on Microtechnologies in Medicine and Biology, Lyon, France, pp. 307-310, 2000. 